\title{
Mirror Approach for the Patients with Unilateral Spatial Neglect and Mirror Agnosia
}

\author{
SATORU WATANABE ${ }^{1,2)}$, KaZU Amimoto ${ }^{2)}$ \\ 1) Rehabilitation Center, Kitasato Institute Medical Center Hospital: 6-100 Arai, Kitamoto, \\ Saitama 364-8501, Japan. TEL +81 48-593-1212 \\ 2) Physical Therapy Program, Health Sciences Course, Graduate School of Tokyo Metropolitan \\ University
}

\begin{abstract}
Mirror Agnosia" is characterized by searching for the mirror image and showing difficulty of modifying behavior even after the object is presented in real space (Ramachandran, 1997). The possibility of therapeutic intervention of using a mirror for unilateral spatial neglect (USN) with mirror agnosia was suggested, but few researches on mirror agnosia and mirror approach for USN have been reported. The purpose of this study was to confirm the influence of the use of a mirror for USN with mirror agnosia. Two patients with left hemiparesis with USN were evaluated. We propped a mirror on the right side of the patient parallel to the sagittal plane and presented a ball apart from it. We asked the patient to grasp it with looking into the mirror. If they could not reach the actual ball, we shifted the ball closer to the mirror and tried again. When two patients grasped the ball, we asked them to reach it repeatedly. Albert test was executed before and after the session. Both patients could not reach the ball initially, but were able to grasp it when it was closer to the mirror. They showed an improvement in the Albert test after this approach. This method might be useful therapeutic intervention for USN with mirror agnosia.
\end{abstract}

Key words: Mirror agnosia, Unilateral spatial neglect, Mirror approach

(This article was submitted Aug. 16, 2006, and was accepted Oct. 10, 2006)

\section{INTRODUCTION}

Ramachandran et al. ${ }^{1)}$ reported four right hemisphere stroke patients with USN who kept searching for the virtual object in a mirror placed on the right side of them and showed difficulty of modifying their behavior even after the object was presented in real space. He called their neurological sign "mirror agnosia", and he suggested in another study the possibility of therapeutic intervention with a mirror for $\mathrm{USN}^{2)}$.

Conventionally, setting a mirror in front of patients with USN for correcting distortion of their posture has been in turn noticed to cause of confusion mentally. In contrast, Ramachandran's method is very interesting from the point of view of placing a mirror parallel to the sagittal plane close to the patients' right side. However, few researches on the mirror approach to USN with mirror agnosia have been reported after his study. Thus, we examined the existence of mirror agnosia in two patients with left USN after right parietal lobe lesion and investigated the influence on USN of the approach using a mirror. The purpose of present study was to investigate whether the approach is available for clinical evaluation and treatment of USN.

\section{SUBJECTS}

\section{Patient 1}

Patient 1 was an 85 year-old female who sustained a right hemisphere hemorrhage.

CT scans revealed damage to the subcortical 
structures of the parietal lobe. She had severe left hemiparesis (Brunnstrom recovery stage: upper $\operatorname{limb}=\mathrm{II}$, finger $=\mathrm{I}$, lower limb=II), severe sensory loss, hyper reflexia, and hypertension, but her right limb was intact. She was alert, and indicated left USN (Behavioral inattention test: $\mathrm{BIT}=38$ points), anosognosia (Bisiach score $=3$ ), pusher syndrome and dementia (Mini-Mental State Examination: MMSE=11). Hemianopia was not present. Left homonymous was not admitted by the sitting face to face method. The time of testing was 35 days post stroke.

\section{Patient 2}

Patient 2 was an 89 year-old female who sustained infarction in the right hemisphere. CT scans revealed damage extending from the temporal to the parietal lobe. She presented with mild left hemiparesis (Brunnstrom recovery stage: upper $\operatorname{limb}=\mathrm{VI}$, finger $=\mathrm{VI}$, lower $\lim b=\mathrm{VI})$. The sense were intact and she was alert. She showed mild left USN (line bisection $=16 \mathrm{~mm}$ shift to rightward, line cancellation $=3$ omissions, $B I T=133$ points), constructional disability and hemianopia, but she did not have remarkable dementia (MMSE=24). The time of testing was 9 days post stroke.

Both patients gave their informed consent to participate in the study.

\section{METHODS}

We propped a mirror up vertically on the right side of the patients who were in a wheelchair, parallel to the sagittal plane. The experimenter stood behind them. They were asked to turn their head and eyes to the right to look the mirror and asked "What is this?"

After we ensured they recognized the mirror as a mirror, we asked what was reflected in the mirror. We asked them to close their eyes and hung a ball of pink color $(6.5 \mathrm{~cm}$ in diameter) about $50 \mathrm{~cm}$ forward and $20 \mathrm{~cm}$ below their eyes $(50 \mathrm{~cm}$ apart from the mirror), so that it was well out of their left neglected visual field. Then, we asked them to open their eyes and asked "Do you find any added object?" If they replied, "a ball", the experimenter added "reach out and grasp the ball, please." If they answered, "I cannot grasp the ball," or reached straight into and kept searching for the mirror, we judged that mirror agnosia was positive. In this case, we asked the patients to close their eyes again and moved the ball closer to the mirror by $10 \mathrm{~cm}$ and again asked them to reach for the ball until they were repeatedly able to grasp the actual ball.

When they were able to grasp the actual ball, we measured the distance of the ball from the mirror and recorded it.

We also moved the ball away from the mirror in $10 \mathrm{~cm}$ increments, and when the patients could not grasp the actual ball, we again asked them to reach for the ball in that position five times.

Moreover, to investigate whether the use of a mirror in this test influenced USN, we performed the Albert test before and after the evaluation.

\section{RESULTS}

Patient 1, when we initially showed her the ball, patted the mirror and claimed, "I cannot grasp it because it is floating in the mirror". She was able to grasp the actual ball when it had been moved to within $10 \mathrm{~cm}$ of the mirror. The patient searched for the ball again when we separated the ball from the mirror by $40 \mathrm{~cm}$. Then, we shifted the ball the mirror by $40 \mathrm{~cm}$ and $30 \mathrm{~cm}$ in turns and asked her to reach the ball. The patient became able to grasp the ball even at distance from the mirror $40 \mathrm{~cm}$ after five repeated trials, and in addition, immediately after that, even at $50 \mathrm{~cm}$ from the mirror she could grasp the actual ball (Fig. 2). She showed 23 omissions on the Albert test before the mirror session and improved to 4 omissions after it.

Patient 2, when initially showed the ball, appealed while pointing at the mirror, "It would be
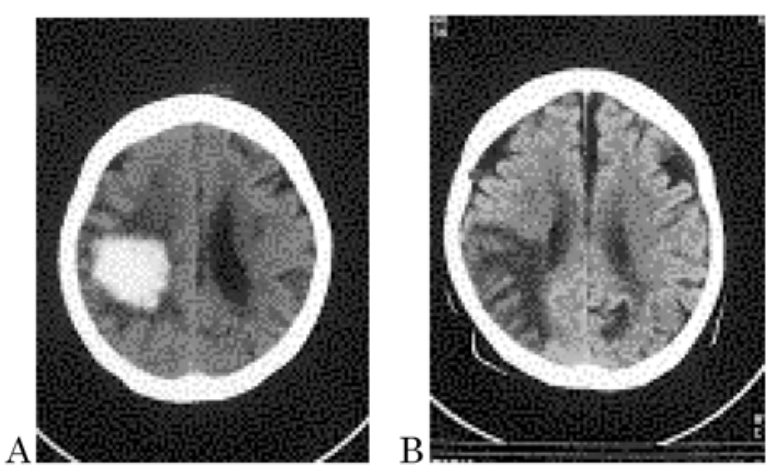

Fig. 1. (A) CT scan image for patient 1. It revealed a high intensity area on the subcortical structures of the right parietal lobe. (B) CT scan image for patient 2 . It revealed a low intensity area extending from the temporal to the parietal lobe. 


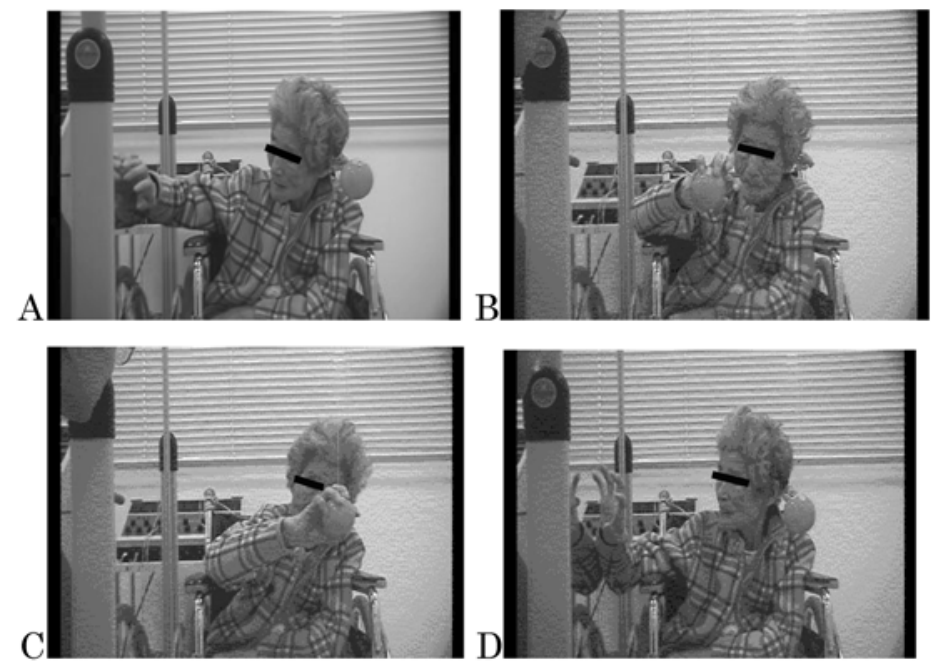

Fig. 2. Behavior of patient 1. (A) When the ball was initially presented, she patted the mirror. (B) She grasped the actual ball when it was $10 \mathrm{~cm}$ from the mirror. (C) Then, she could grasp the ball it was until separated $30 \mathrm{~cm}$ from the mirror. (D) But she could not grasp the actual ball again at $40 \mathrm{~cm}$ from the mirror.

not better to be able to take it?" She was able to grasp the actual ball when it was only $5 \mathrm{~cm}$ from the mirror, and she could reach for the actual ball without hesitating when it was separated to $50 \mathrm{~cm}$. She showed it 2 omissions on the Albert test before the mirror session and improved to 0 omission after it.

\section{DISCUSSION}

Both patients 1 and 2 had their engaged attention by the mirror image, and they could not reach the actual ball when it was presented in their left space in first trial. When we moved the ball close to the mirror in increments and the ball was placed within their visual field, they found the ball as if they had not noticed it until that time, but they were not caught by the mirror image after that. Moreover, the amelioration was shown in the conventional neglect test.

For the reason why their attention was caught by the mirror image, Ramachandran ${ }^{2)}$ described that the syndrome may be a specific consequence of the neglect, or the subtle deficits in spatial abilities that occur following parietal lobe lesions, because when the target appeared just behind and above the patient's right shoulder and the mirror was placed in front of them (in the coronal plane), the patient looked into the mirror but reached straight behind her to grasp the actual target. Beis ${ }^{3)}$ suggested that hemineglect patients experience difficulty in generating new spatial representations due to their inability to adapt the perception-attentional procedures necessary to manipulate mirror-image information.

In the present cases, the patients showed higher brain dysfunction as USN, hemianopia, anosognosia, or cognitive dysfunction as dementia, so their multiple deficits influenced the processing of images. However, the results of the present study suggest that mirror agnosia is a syndrome independent from USN, because the patients showed said "the ball is behind the mirror" and looked behind the mirror frame without searching for the mirror image whereas they initially recognized it as a mirror. Also they paid attention to the actual ball presented in left space after noticing it. When we showed the reverse side of the mirror frame to the patients they did not reach to the mirror frame at all but directly to the actual ball within their visual field. In another case, a patient with left USN could reach the actual ball without responding to the mirror image in the same procedure ${ }^{4)}$. In addition, the patients reported by Ramachandran showed intelligence and no hint of dementia ${ }^{1)}$.

As USN was improved, it is possible to consider 
that the effect was due to visuo-motor stimulation shifting the target to the left side gradually by optokinetic stimulation $^{5)}$. Nevertheless, the results of the present study might be expected as an immediate effect of short term intervention, since the attention to the left side was improved right after noticing the actual ball. However, continuity of the effect was temporal, up to the next day in our cases.

The subjects of our study were only two patients, so we have insufficient data to discuss the mechanism of mirror agnosia and the mirror method of treatment for USN. In addition, they showed different reactions to mirror approach. More detailed research of many cases is required.

\section{REFERENCES}

1) Ramachandran VS, Altschuler EL, Hillyer S: Mirror agnosia. Proc Biol Sci, 1997, 264: 645-647.

2) Ramachandran VS, Altschuler EL, Stone L, et al.: Can mirrors alleviate visual hemineglect? Med Hypotheses, 1999, 52: 303-305.

3) Beis JM, Andre JM, Barre A, et al.: Mirror images and unilateral spatial neglect. Neuropsychologia, 2001, 39: 1444-1450.

4) Binkofski F, Buccino G, Dohle C, et al.: Mirror agnosia and mirror ataxia constitute different parietal lobe disorders. Ann Neurol, 1999, 46: 51-61.

5) Pizzamiglio L, Frasca R, Guariglia C, et al.: Effect of optokinetic stimulation in patients with visual neglect. Cortex, 1990, 26: 535-540. 